\title{
Méthodologie de la détection de la radioactivité dans l'environnement*
}

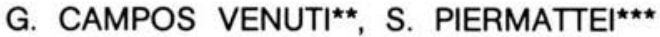 \\ (Manuscrit reçu le 21 décembre 1987)
}

\begin{abstract}
RÉSUMÉ
L'accident de Tchernobyl a mis en évidence, à nouveau, la nécessité de disposer de réseaux de contrôle de la radioactivité artificielle dans l'environnement. Ces réseaux doivent aussi être capables d'affronter des états d'urgence portant sur une grande surface, produits par des accidents qui pourraient survenir à l'intérieur du territoire national, comme à l'extérieur. II s'agit de compléter les réseaux existants, qui fonctionnent en conditions normales, par des systèmes et des protocoles d'intervention à adopter lors de situations exceptionnelles.

Cet article décrit l'organisation actuelle en Italie, et précise quel est le développement déjà planifié des systèmes de surveillance. Nous abordons, en particulier, la question des réseaux nationaux et régionaux en cours de mise en place.
\end{abstract}

\section{ABSTRACT}

The Chernobyl accident has stressed the need of networks for monitoring artificial radioactivity in the environment. These networks must also be able to cope with wide-scale emergencies, connected with accidents inside or outside the national territory. The goal is to supplement the existing networks, which operate in normal conditions with systems and operational directives to be adopted under exceptional circumstances.

This report wants to show the present italian organization and the planned evolution of monitoring systems. A particular focus is on the role of national and regional networks in the making.

* Communication présentée lors du congrès franco-italien SFRP-Associazione italiana di protezione contro le radiazioni, Castelgandolfo (Italie), 12-13 octobre 1987.

** Istituto superiore di sanità, Viale Regina Elena 299, 00161 Roma.

*** Comitato nazionale per la ricerca e lo sviluppo dell' energia nucleare e delle energie alternative, Direzione Sicurezza e protezione (ENEA, DISP), Via Brancati 48, 00144 Roma. 


\section{INTRODUCTION}

La publication $n^{\circ} 43$ de la CIPR [6] présente les éléments méthodologiques du contrôle de la radioactivité de l'environnement, dans les conditions normales; elle définit deux types de surveillance, l'un relatif à la source, l'autre concernant l'individu.

Les figures 1 et 2 présentent la surveillance relative à la source; elles donnent les objectifs à atteindre et les paramètres qui la caractérisent. II faut souligner que les caractéristiques de la source et de son environnement immédiat définissent les voies critiques, objet de la recherche, et que le contrôle s'effectue par une série d'analyses portant sur les composantes des aliments et de l'environnement. Les résultats de ces analyses conduisent à l'estimation de l'équivalent de dose efficace, au niveau du groupe le plus exposé de la population. On effectue des révisions périodiques des pro-

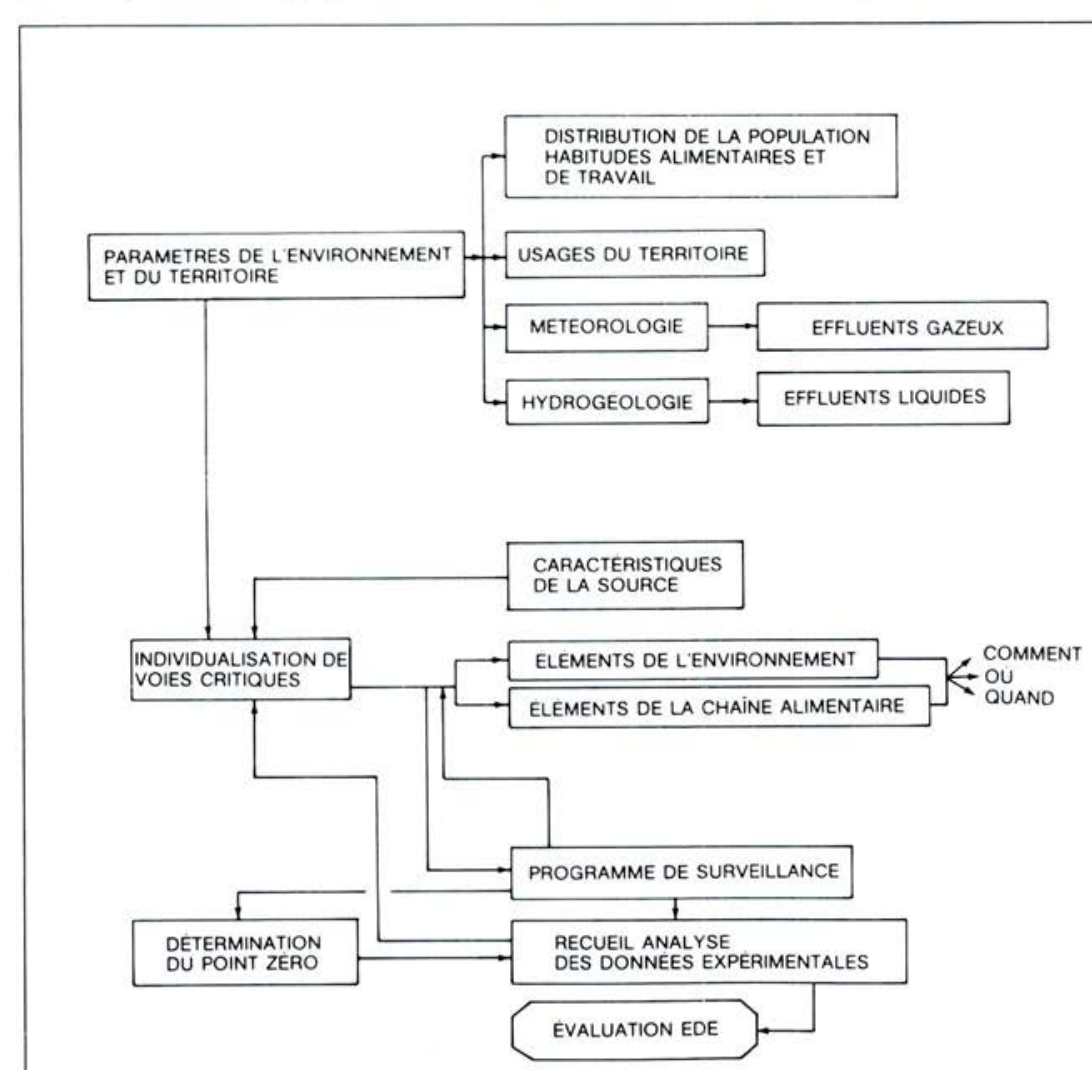

Fig. 1. - Caractérisation de la surveillance concernant la source. 
MÉTHODOLOGIE DE LA DÉTECTION DE LA RADIOACTIVITÉ DANS L'ENVIRONNEMENT
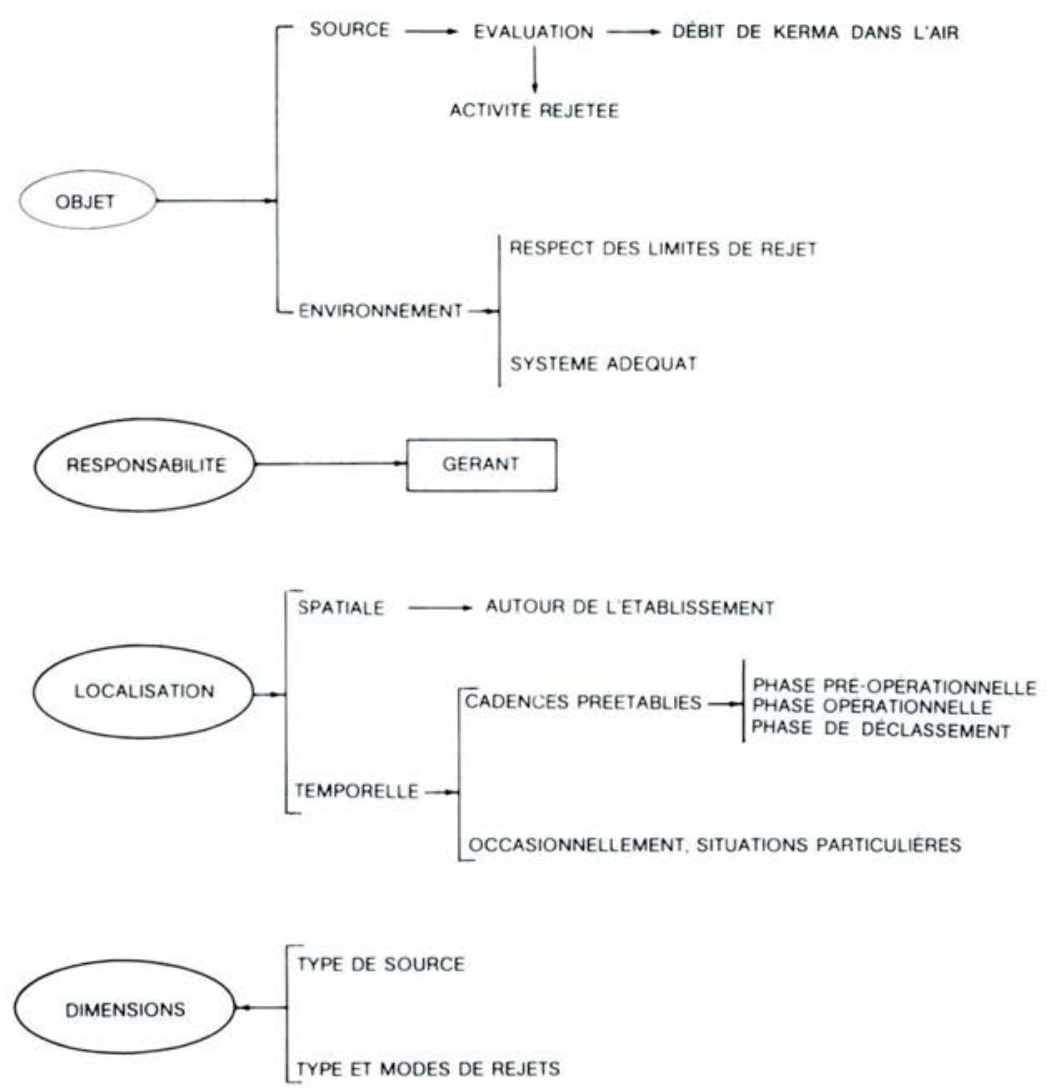

Fig. 2. - Surveillance concernant la source.

grammes de surveillance à la lumière des résultats obtenus; ces programmes ont été vérifiés par de longues années d'utilisation; leur structure a été codifiée.

Dans le domaine des concepts, la CIPR a fait un grand pas en avant, en individualisant les réseaux concus en fonction de l'individu; leur but est d'évaluer l'impact global sur les individus de toutes les sources de rayonnement naturelles et artificielles. L'estimation de l'exposition dépend de la connaissance d'une série de données, englobant territoire et population, et VOL. $23 \cdot \mathrm{N}^{\circ} 2$ 
de la mise au point d'un modèle de transfert de la radioactivité (fig. 3 et 4). La publication $n^{\circ} 43$ attache une importance particulière à l'évaluation de l'exposition provenant des sources naturelles de radioactivité modifiées par les activités humaines, étant donné leur importance au plan sanitaire. Enfin, cette publication souligne que l'élément essentiel de toute surveillance est le contrôle de la qualité, assuré par des mesures reproductibles et comparables. Un programme de contrôle de qualité comporte deux phases:

1. le contrôle de la qualité de l'équipement et des techniques de mesure;

2. la normalisation des méthodes de prélèvement, de préparation des échantillons (par la mise en œuvre de protocoles appropriés), et de la présentation des résultats.

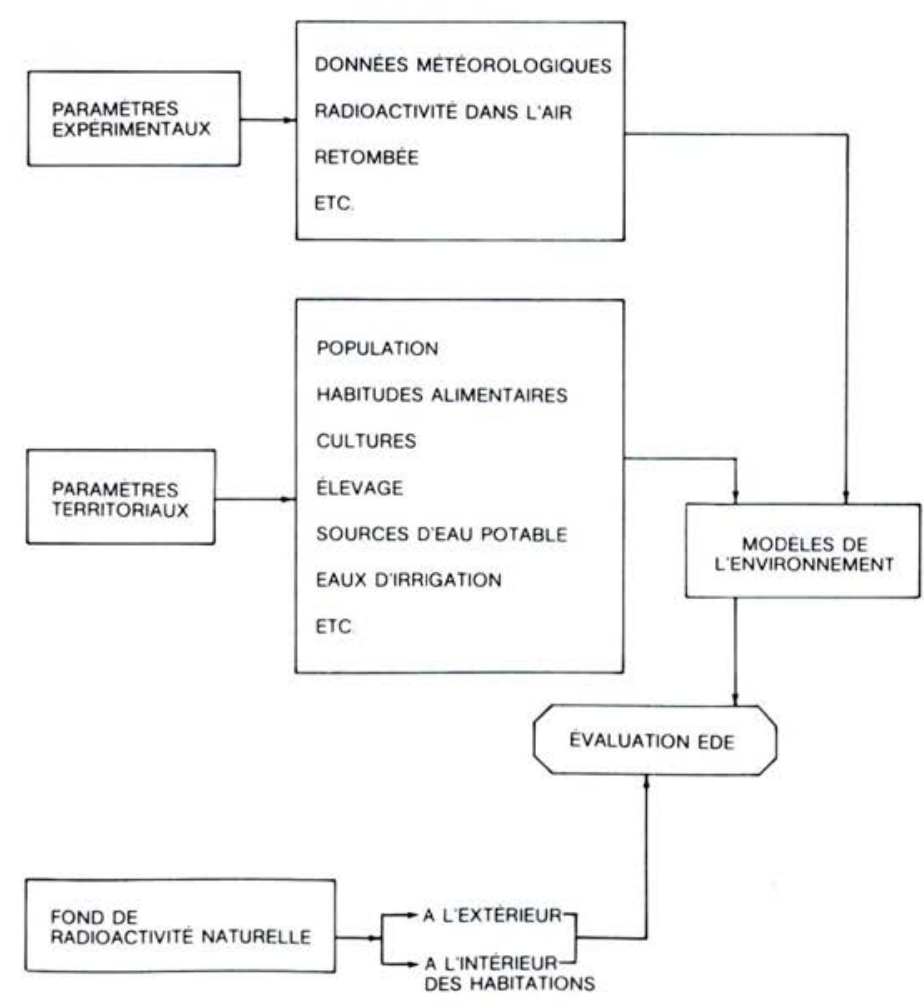

Fig. 3. - Caractérisation de la surveillance concernant les individus. 


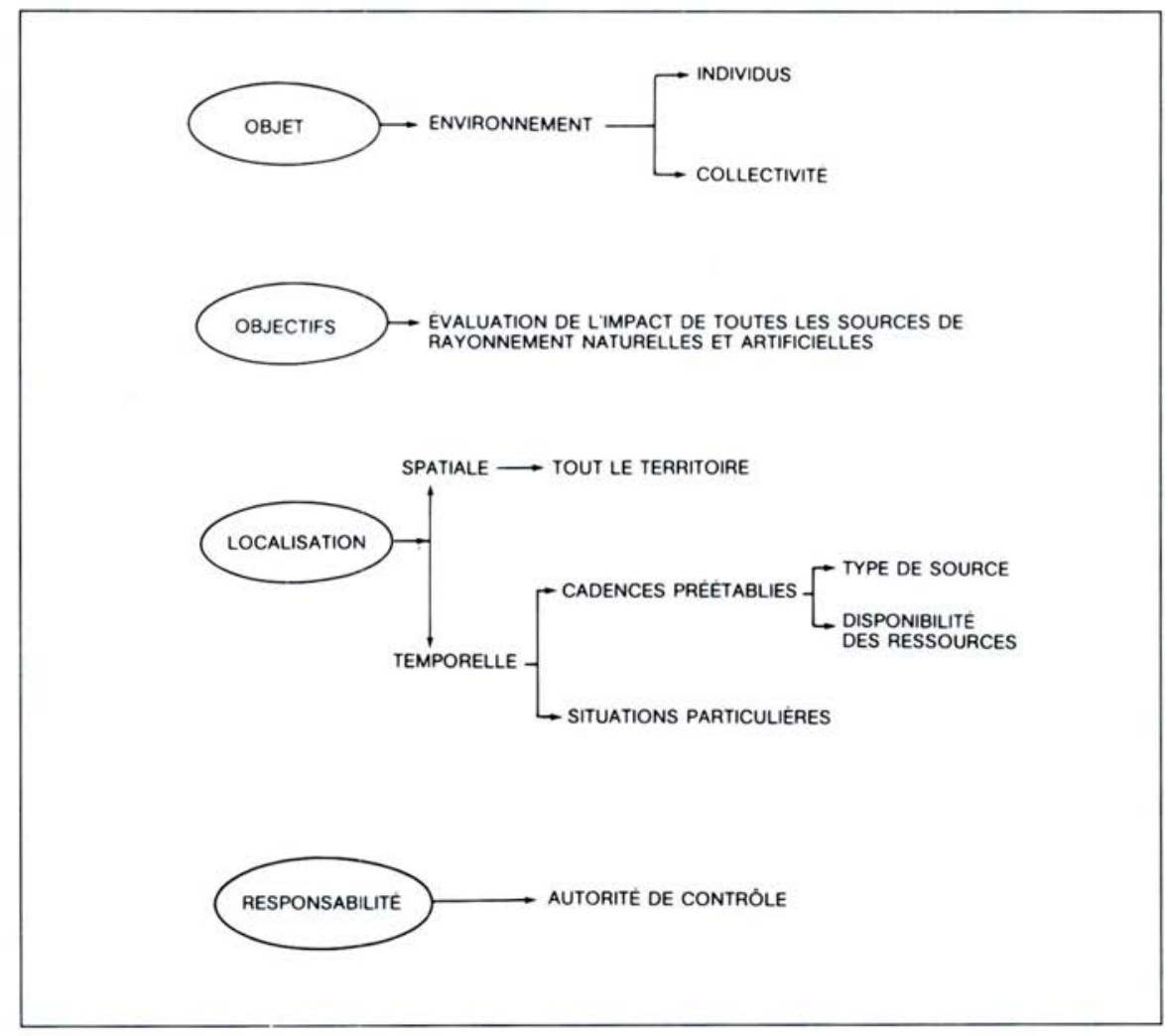

Fig. 4. - Surveillance concernant les individus.

\section{SITUATION, EN ITALIE, \\ des RÉseauX de surVEILLANCE LIÉs À L'INDIVIDU (fig. 5).}

Ces réseaux furent mis en place en Italie vers le milieu des années cinquante, quand les explosions nucléaires dans l'atmosphère attirèrent l'attention des gouvernements sur le problème de la radioactivité dans l'environnement, et sur son importance sanitaire.

Les premières mesures portèrent sur l'activité atmosphérique, dont on déterminait l'activité $\beta$ totale [4]. Dans quelques stations, quelques années après, ces mesures furent complétées par de la spectrométrie gamma. Les analyses de la concentration atmosphérique furent suivies de celles d'autres composantes de l'environnement : retombées, eaux de surface, constituants de la chaîne alimentaire comme le lait, la viande, les poissons, les végétaux, les produits industriels alimentaires et, en 1983, certains régimes alimentaires. 


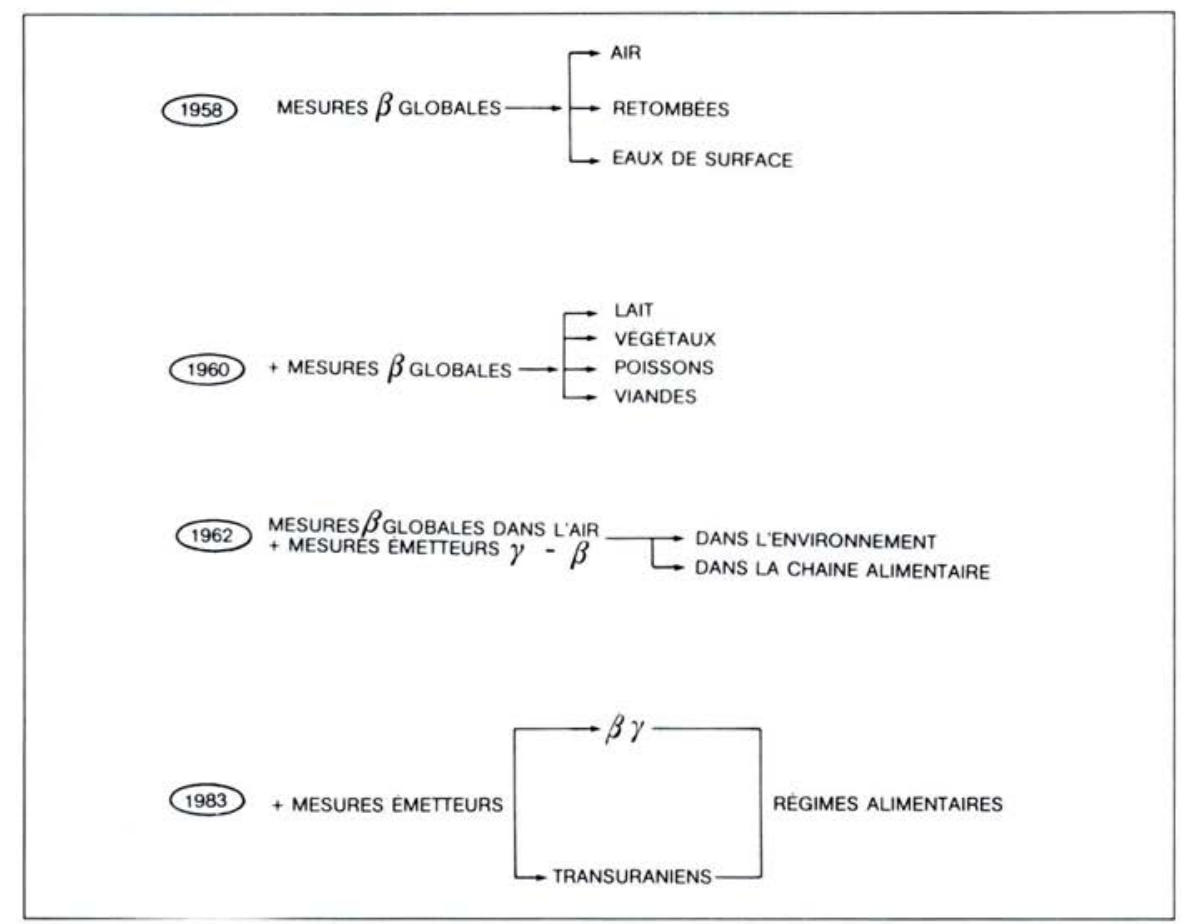

Fig. 5. - Réseaux nationaux: évolution chronologique.

\section{RÉVISION DES RÉSEAUX DE SURVEILLANCE LIÉS À L'INDIVIDU}

Dans presque tous les pays, l'accident de Tchernobyl a fait apparaître, de manière urgente, l'importance des réseaux de surveillance de l'environnement. Les organismes de contrôle ont ainsi été poussés à réviser les critères de base qui sous-tendent les objectifs de ces réseaux. Dans un tel cadre, il a fallu relire, de manière critique, la publication $n^{\circ} 43$ de la CIPR.

La contamination extrêmement étendue du territoire, par l'accident de Tchernobyl, a démontré la nécessité de structurer les réseaux liés à l'individu, afin qu'ils puissent faire face aux urgences couvrant de grandes surfaces. II faut donc créer un réseau de surveillance de l'activité atmosphérique et des données météorologiques de base, qui couvre le territoire. Ce réseau devra permettre de mesurer, en temps réel, les concentrations dans l'air de radionucléides artificiels qui, bien que n'ayant pas une importance suffisante pour exiger des interventions immédiates et urgentes, pourraient fournir une indication de l'existence de situations anormales. La surveillance de l'air en temps réel devra être complétée par un système de mesure de la radioactivitè à haute altitude et au sol, installé sur des avions; on pourra ainsi tracer 
des cartes de la contamination sur de vastes zones, en un laps de temps assez bref.

Si l'on ajoute au réseau de surveillance, un réseau de données pluviométriques et de mesure des retombées radioactives, réparti sur des sites météorologiquement représentatifs, on obtiendra les données d'entrée d'un modèle d'environnement pouvant apporter des réponses rapides; il permettra alors de planifier les échantillonnages de façon pertinente.

En outre, il faut souligner que chaque élément important de la chaîne alimentaire, ainsi que certains indicateurs de l'environnement, doivent posséder leurs réseaux d'échantillonnage, qui pourront être différents selon les saisons, mais en nombre suffisant pour couvrir les sites représentatifs de l'ensemble du pays et être déclenchés en cas de nécessité. Sous peine de rendre vains les efforts, il est fondamental que les techniques d'échantillonnage soient normalisées, et que la possibilité d'initiatives personnelles soit réduite à sa valeur minimale. II est important de mettre au point des méthodes rapides d'individualisation des émetteurs $\beta$ et des transuraniens.

En ce qui concerne la contamination des aliments, il faut mettre au point, à l'échelle internationale, les niveaux de référence à utiliser pour réglementer l'emploi et la circulation des denrées à la suite d'un accident nucléaire ou d'une augmentation anormale de la radioactivité. II faut, de plus, réclamer la création de modèles radioécologiques qui soient validés au niveau supranational.

A la suite de l'accident de Tchernobyl, on a attaché une importance considérable à l'échange d'informations entre les pays et la transmission des données de mesure, ainsi qu'au problème très complexe de l'information des populations. Dans ces domaines, il faudra faire des efforts au niveau international pour couvrir les aspects essentiels de l'organisation (par exemple, la mise au point de procédures codifiées ${ }^{\star}$ ).

\section{RENFORCEMENT DES RÉSEAUX NATIONAUX}

Avant d'aborder cette question, nous allons rappeler quelle est la législation italienne sur le contrôle de la radioactivité artificielle.

Dans notre pays, l'une des tâches principales du ministère de la Santé [2] est, depuis 1964, le contrôle de la radioactivité de l'environnement et de la chaîne alimentaire. Pour réaliser ces contrôles, des réseaux nationaux de mesure ont été mis en place; ils sont coordonnés par l'ENEA DISP. Depuis 1972 [1] le contrôle de la radioactivité de l'environnement a été délégué (mais pas transféré) aux autorités régionales; ce n'est que l'année dernière que le ministère a donné aux organismes régionaux des directives pour la mise en œuvre de ces contrôles; il prévoyait aussi la création, dans chaque région, d'un laboratoire mesurant la radioactivité de l'environnement. Certaines régions disposaient d'un tel laboratoire; d'autres l'ont créé durant l'état d'urgence de Tchernobyl; plusieurs centres de mesure sont en cours de réalisation. Au niveau régional, on a prévu des réseaux de surveillance à fonction double: il s'agit de réseaux liés à l'individu car ils doivent évaluer et

(*) On pourra, dans ce domaine, consulter les notifications publiées par l'AIEA et par la CEE (NdR)

VOL. $23 \cdot \mathrm{N}^{\circ} 2$ 
suivre la situation radiologique globale sur tout le territoire; ce sont aussi des réseaux liés à la source, car ils doivent contrôler l'impact des sources radioactives existant dans la région.

Après l'accident de Tchernobyl (fig. 6), on a mis en œuvre le renforcement du réseau national, grâce à la contribution des laboratoires régionaux fonctionnant déjà. Pour ce renforcement, le critère suivi concernant l'environnement $[5,7]$, est d'aboutir rapidement à la couverture homogène du territoire; en ce qui concerne la chaîne alimentaire, le critère est le contrôle des principaux centres de production et de consommation.

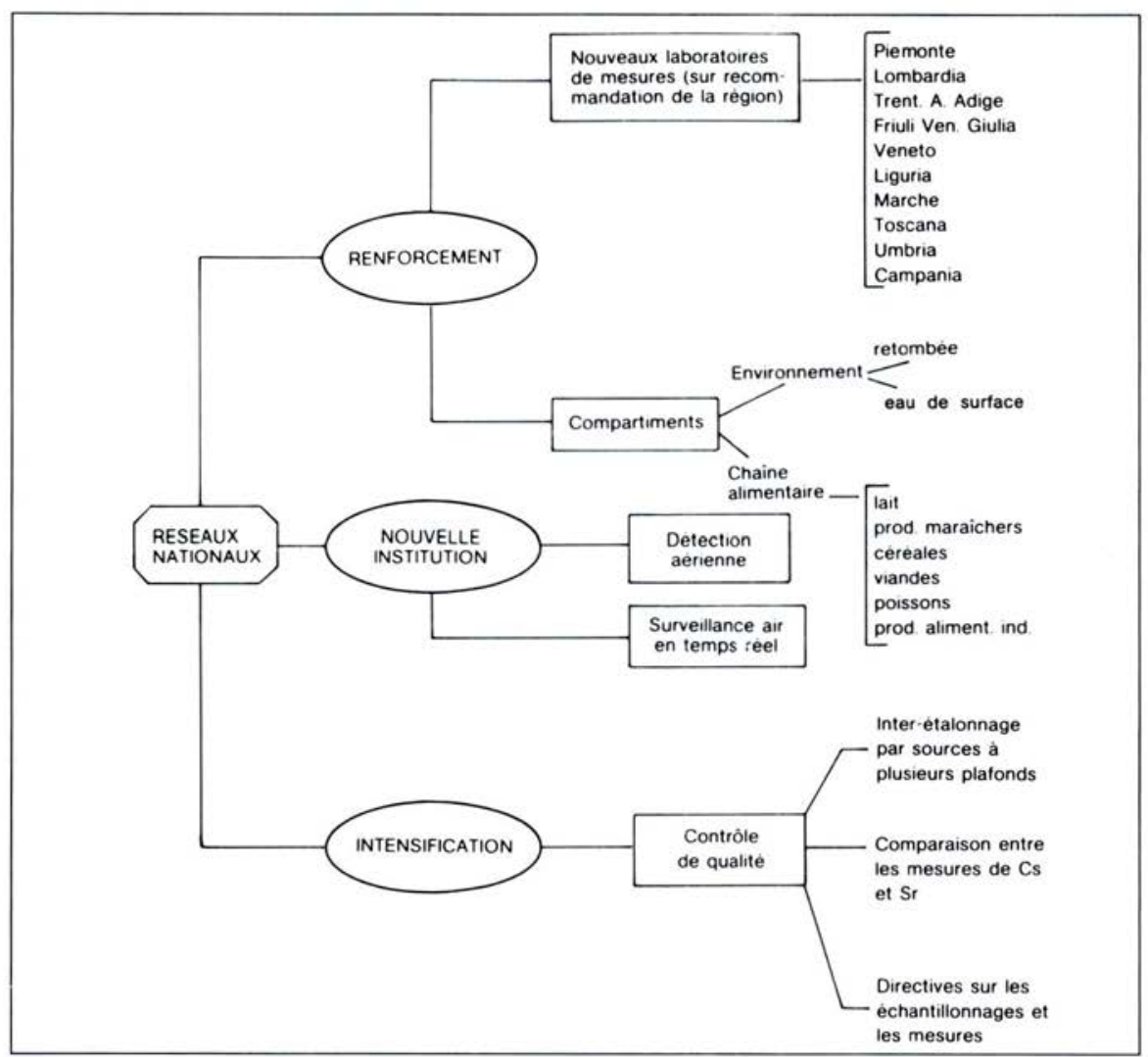

Fig. 6. - Réseaux nationaux: évolution après Tchernobyl.

Soulignons l'importance grandissante que revêt la normalisation des échantillonnages et des mesures, au fur et à mesure qu'augmente le nombre des laboratoires. Dans cette optique, nous avons effectué une intercomparaison des mesures de ${ }^{90} \mathrm{Sr}$ et ${ }^{137} \mathrm{Cs}$ dans le lait, et nous avons préparé le protocole d'échantillonnage. Pour ce qui est de la radioactivité naturelle, le problème n'a été abordé, en Italie, que sous l'aspect d'études et 
de recherches. Le moment est venu d'aborder ce problème de manière systématique, au niveau du territoire national, dans les lieux de travail (établissements thermaux, mines, etc.), comme à l'intérieur des habitations. Quelques laboratoires régionaux, répartis de manière homogène sur le territoire, pourront organiser et mener à leur fin les campagnes de mesure nécessaires. Cela demandera, au niveau central, l'établissement des méthodologies à employer dans les mesures de routine; au niveau international, il faudra fixer les niveaux de référence auxquels les résultats puissent être confrontés.

Enfin, rappelons-le, les directives du ministère de la santé, que nous venons de mentionner, prévoient une commission de coordination nommée par le ministère lui-même, qui aura pour tâches:

a) de tenir compte des procédés de mesure mis en place par chaque région, en relation avec les impératifs et les conditions locales;

b) d'examiner les résultats de mesure fournis par les régions;

c) de susciter la mise à jour des procédés de mesure, et d'assurer un service de consultation.

\section{MODĖLES DE TRANSFERT DE :A RADIOACTIVITÉ DANS L'ENVIRONNEMENT}

Après l'accident de Tchernobyl, on a vérifié la nécessité de disposer de modèles de transfert, et leur importance. II faut orienter dans cette direction les efforts nationaux et internationaux pour fournir aux réseaux de surveillance de la radioactivité dans l'environnement un bon instrument de décision.

Comme on le sait, on a élaboré de nombreux modèles, qui sont maintenant disponibles. La plupart des modèles évaluant le risque sanitaire dû au rejet de radionucléides dans l'environnement impliquent une situation stationnaire, ou quasi stationnaire, entre les différents compartiments. Cela signifie que la quantité des radionucléides existant dans un compartiment est corrélable à celle existant dans un autre, au moyen d'un facteur de conversion (par exemple: la relation entre le dépôt au sol et la concentration dans les végétaux).

Les modèles stationnaires ne sont pas applicables aux rejets de courte durée, ni aux radionucléides à vie longue, car il faut tenir compte de la variation dans le temps du processus. On a assisté à un nouvel essor de la mise au point de modèles dynamiques essayant de décrire de la manière la plus réaliste possible la cinétique réelle des phénomènes. Le domaine d'application des modèles dynamiques est vaste: ils peuvent s'appliquer à n'importe quel genre de rejet. Dans le cas le plus simple, la cinétique de ces modèles est du premier ordre: les paramètres d'entrée et (ou) de sortie de chacun des compartiments composant le modèle sont exprimés par le produit d'une constante (facteur de transfert) par la concentration du radionucléide considéré dans ce compartiment. II est donc possible d'obtenir des solutions analytiques. Le passage de la théorie à la réalité implique de remplacer la constante de transfert par une fonction du temps qui ne conduit pas à des solutions analytiques, mais à des solutions numériques corrélées au développement d'un code de calcul. 
Soulignons que la variation en fonction du temps des facteurs peut être liée à de nombreux paramètres comme la saison, le type d'agriculture pratiqué, le milieu biologique, la répartition, la nature, la richesse des divers éléments importants de l'environnement, etc. Soulignons aussi que la cinétique des processus peut dépendre de la distance du point de rejet. Même dans le cas des modèles dynamiques, il existe donc des limitations intrinsèques de leur développement, liées à la difficulté de quantifier correctement tous les paramètres importants (le nombre de mesures nécessaires devient prohibitif). Pour cette raison, et parce que tout modèle n'est qu'une représentation abstraite de la réalité, on a tendance à développer le modèle en tenant compte des paramètres qui sont déterminables expérimentalement. Dans la majeure partie des cas, ces paramètres représentent un ensemble de processus, et le modèle permettra de prévoir les situations qui se rapprochent le plus possible de celles dont les paramètres ont été mesurés.

Les points fondamentaux sont donc:

a) l'estimation de la fiabilité des prévisions faites grâce à un modèle d'environnement;

b) l'efficacité du modèle lui-même.

Un rapport de l'Agence internationale pour l'énergie atomique (AIEA) [3] aborde le problème de la fiabilité. Ce rapport analyse les diverses phases qui interviennent dans la réalisation d'un modèle, afin d'en étudier l'impact sur sa fiabilité : individualisation d'un scénario, choix du modèle, formation du code de calcul, traitement des résultats et leur présentation. On doit porter une attention particulière aux paramètres d'entrée, qui constituent simplement l'ensemble des variables intervenant dans les équations décrivant le modèle. Ces paramètres devront être déduits de mesures faites dans des conditions aussi semblables que possible à celles auxquelles le modèle s'applique. Un choix erroné des paramètres fausse, dans tous les cas, les prévisions du modèle.

En général, pour les divers radionucléides et les diverses voies considérées, on ne dispose pas d'un grand nombre de données expérimentales, permettant de quantifier les paramètres en fonction du temps, de l'espace, ou des différentes techniques agricoles. Toutefois, on assiste à une intense activité de recherche dans presque tous les pays qui cherche à déterminer les paramètres de transfert de la radioactivité dans l'environnement, dans diverses conditions expérimentales.

Soulignons enfin qu'on ne doit considérer un modèle comme valable qu'après qu'il ait été contrôlé de manière adéquate, la comparaison des prévisions aux observations expérimentales assurant un niveau acceptable de précision, à déterminer à chaque fois.

\section{REMERCIEMENTS}

Les auteurs remercient Mlle Paola DI CIACCIO pour son travail de traduction, MIle Simona CECCARELLI pour sa contribution technique. 


\section{RÉFÉRENCES}

[1] cfr. DPR 4/72, 1972, DPR 616/77, 1977, Legge 833/78, 1978.

[2] DPR 13 février 1964, n. 185, Sicurezza degli impianti e protezione sanitaria dei lavoratori e delle popolazioni contro li pericoli delle radiazioni ionizzanti derivanti dall'impiego pacifico dell'energia nucleare, 1964.

[3] AGENCE INTERNATIONALE POUR L'ÉNERGIE ATOMIQUE (AIEA). Procedure for evaluating the reliability of predictions made by environmental transfer models (Safety Series) Vienne: AIEA, à paraître.

[4] ENEA. Rapporti annuali sulla radioattività ambientale in Italia. Rome: ENEA.

[5] ENEA-DISP. Incidente di Chernobyl: conseguenze radiologiche in Italia, relazione al $27 / 5 / 1986$. DOC/DISP (86) $14,1986,1-50$.

[6] INTERNATIONAL COMMISSION ON RADIOLOGICAL PROTECTION (ICRP). Environmental monitoring for the radiation protection of the population (ICRP Publication 43). Oxford: Pergamon press, 1984.

[7] ISTITUTO SUPERIORE DI SANITẢ (ISS). ॥ rischio ambientale nella produzione di energia: risultati sperimentali, calcoli e riflessioni dopo Chernobyl. Ann. ISS, 1987 , 23 (2) $177-252$. 